Article

\title{
Generalized Integral Transforms via the Series Expressions
}

\section{Hyun Soo Chung}

Department of Mathematics, Dankook University, Cheonan 31116, Korea; hschung@dankook.ac.kr

Received: 25 February 2020; Accepted: 2 April 2020; Published: 6 April 2020

\begin{abstract}
From the change of variable formula on the Wiener space, we calculate various integral transforms for functionals on the Wiener space. However, not all functionals can be obtained by using this formula. In the process of calculating the integral transform introduced by Lee, this formula is also used, but it is also not possible to calculate for all the functionals. In this paper, we define a generalized integral transform. We then introduce a new method to evaluate the generalized integral transform for functionals using series expressions. Our method can be used to evaluate various functionals that cannot be calculated by conventional methods.
\end{abstract}

Keywords: generalized integral transform; kernel; Wiener-Itô-Chaos expansion; Riesz's theorem; Hahn-Banach theorem

MSC: Primary 60J65; Secondary 28C20

\section{Introduction}

For a positive real number $T$, let $C_{0}[0, T]$ be the space of all real-valued continuous functions $x$ on $[0, T]$ with $x(0)=0$. Let $\mathcal{M}$ denote the class of all Wiener measurable subsets of $C_{0}[0, T]$ and let $m$ denote Wiener measure. Then, as is well-known, $\left(C_{0}[0, T], \mathcal{M}, m\right)$ is a complete measure space. Let $K \equiv K_{0}[0, T]$ be the space of complex-valued continuous functions defined on $[0, T]$ which vanish at $t=0$. We denote the Wiener integral of a Wiener integrable functional $F$ by

$$
\int_{C_{0}[0, T]} F(x) d m(x)
$$

In a unifying paper [1], Lee introduced an integral transform of analytic functionals. For certain complex numbers $\gamma$ and $\beta$ and for certain classes of functionals, the (modified) Fourier-Wiener transform, the Fourier-Feynman transform and the Gauss transform are special cases of Lee's integral transform $\mathcal{F}_{\gamma, \beta}$ defined by the formula

$$
\mathcal{F}_{\gamma, \beta}(F)(y)=\int_{C_{0}[0, T]} F(\gamma x+\beta y) d m(x), \quad y \in K,
$$

see [2-12]. They have established various fundamental formulas and relationships involving the convolution product, first variation (derivative), translation theorem and Cameron-Strovick theorem. However, the study of integral transform has been limited to classes of functionals like the Banach algebra $[7,8]$ of the form

$$
F(x)=\int_{L_{2}[0, T]} \exp \{\langle w, x\rangle\} d f(w)
$$


where $\langle w, x\rangle$ is the Paley-Wiener-Zygmund (PWZ) stochastic integral and $f$ is a complex measure on the Borel $\sigma$-algebra $\mathcal{B}\left(L_{2}[0, T]\right)$, a class $\mathbb{E}_{0}$ of functionals [5] of the form

$$
F(x)=f(\langle w, x\rangle)
$$

where $f$ is an appropriate function on $\mathbb{C}$ or tame (basic) functionals $[6,9,10,12]$ of the form

$$
F(x)=f\left(x\left(t_{1}\right), \cdots, x\left(t_{n}\right)\right)
$$

where $0=t_{0}<T_{1}<\cdots<t_{n}=T$ is a partition of $[0, T]$. For many functionals it is difficult or impossible to calculate the generalized integral transform via the change of variable formula on the Wiener space. For example, the following Wiener integral, which appears in calculation of generalized integral transform,

$$
\int_{C_{0}[0, T]} \sin (x(T)) d m(x)
$$

is not easy and the calculation of Wiener integral

$$
\int_{C_{0}[0, T]} \frac{1}{1+x^{2}(T)} d m(x)
$$

is impossible. Furthermore the following Wiener integral

$$
\int_{C_{0}[0, T]} x(t) \sin (x(T)) d m(x)
$$

is harder to calculate (for more detailed see Section 3 below). Many attempts have been made to solve these problems.

In this paper, we introduce a new generalized integral transform that contains a kernel in its definition. We apply the Taylor series expansion in Euclidean space and the results and formulas in functional analysis to obtain formulas for the generalized integral transform. Finally, we evaluate the integral transform of several functionals, including Equation (2). Many functionals cannot be calculated by conventional methods. We use our method to evaluate them. We expect that, by providing a calculation method, more functionals can be calculated.

\section{Preliminaries and Definitions}

In this section, we state some definitions and notations to understand this paper. We then define a new generalized integral transform and we then give simple examples.

A subset $B$ of $C_{0}[0, T]$ is said to be scale-invariant measurable provided $\rho B$ is $\mathcal{M}$-measurable for all $\rho>0$. For a scale-invariant measurable set $N$ is said to be a scale-invariant null set provided $m(\rho N)=0$ for all $\rho>0$. Furthermore, a property that holds except on a scale-invariant null set is said to hold scale-invariant almost everywhere (s-a.e.) [13].

For $v \in L_{2}[0, T]$ and $x \in C_{0}[0, T]$, let $\langle v, x\rangle$ denote the PWZ stochastic integral $[1,2,4]$. Then we have the following assertions.

(i) For each $v \in L_{2}[0, T],\langle v, x\rangle$ exists for a.e. $x \in C_{0}[0, T]$.

(ii) If $v \in L_{2}[0, T]$ is a function of bounded variation on $[0, T],\langle v, x\rangle$ equals the Riemann-Stieltjes integral $\int_{0}^{T} v(t) d x(t)$ for s-a.e. $x \in C_{0}[0, T]$.

(iii) The PWZ stochastic integral $\langle v, x\rangle$ has the expected linearity property.

(iv) The PWZ stochastic integral $\langle v, x\rangle$ is a Gaussian process with mean 0 and variance $\|v\|_{2}^{2}$.

We are now ready to state the definition of the generalized integral transform of functionals on $K$. 
Definition 1. Let $\gamma$ and $\beta$ be nonzero complex numbers. Let $F$ and $G$ be functionals defined on $K$. The generalized integral transform $T_{\gamma, \beta}^{G}(F)$ of $F$ given $G$ is defined by the formula

$$
T_{\gamma, \beta}^{G}(F)(y)=\int_{C_{0}[0, T]} F(\gamma x+\beta y) G(x) d m(x), \quad y \in K
$$

if it exists. In this case, the functional $G$ is called the kernel of generalized integral transform.

In our next remark, we shall explain the usefulness of generalized integral transform $T_{\gamma, \beta}^{G}$.

Remark 1. (1) Consider a differential equation

$$
\frac{\partial}{\partial t} \psi(u, t)=\frac{1}{2} \Delta \psi(u, t)-V(u) \psi(u, t)
$$

with the initial condition $\psi(u, 0)=\varphi(u)$, where $\Delta$ is the Laplacian and $V$ is a potential function [14]. Using the Feynman-Kac formula, we know that the solution of Equation (4) can be written as a Wiener integral

$$
\int_{c_{0}[0, T]} \exp \left\{-\int_{0}^{T} V(x(s)) d s\right\} \varphi(x(T)) d m(x) .
$$

It looks like that $\int_{\mathcal{C}_{0}[0, T]} F(x) G(x) d m(x)$. This tells us that our integral transform is a worthy subject to study.

(2) When $G \equiv 1$ on $K, T_{\gamma, \beta}^{1}(F)(y)=\mathcal{F}_{\gamma, \beta}(F)(y)$ for each $y \in K$. That is to say, it is the integral transform of the functional $F$. This means that all formulas and results in previous papers [4-12] are corollaries of our results and formulas in this paper.

We state a well-known integration formula which is used later in this paper.

Theorem 1. Let $\left(t_{1}, \cdots, t_{n}\right)$ be an n-tuple of $[0, T]$ with $0=t_{0}<t_{1}<\cdots<t_{n}=T$. Let $f: \mathbb{R}^{n} \rightarrow \mathbb{C}$ be Lebesgue measurable and let

$$
F(x)=f\left(x\left(t_{1}\right), \cdots, x\left(t_{n}\right)\right)
$$

Then

$$
\begin{aligned}
& \int_{C_{0}[0, T]} F(x) d m(x) \\
& =\int_{C_{0}[0, T]} f\left(x\left(t_{1}\right), \cdots, x\left(t_{n}\right)\right) d m(x) \\
& =\left(\prod_{j=1}^{n} \frac{1}{2 \pi\left(t_{j}-t_{j-1}\right)}\right)^{\frac{1}{2}} \int_{\mathbb{R}^{n}} f(\vec{u}) \exp \left\{-\sum_{j=1}^{n} \frac{\left(u_{j}-u_{j-1}\right)^{2}}{2\left(t_{j}-t_{j-1}\right)}\right\} d \vec{u}
\end{aligned}
$$

in the sense that if either side of (5) exists, then both sides exist and the equality holds.

We give simple examples to illustrate the usefulness of the kernel for the generalized integral transform, and to explain some differences between the generalized integral transform $T_{\gamma, \beta}^{G}$ and the integral transform $\mathcal{F}_{\gamma, \beta}$. 
Example 1. Let the kernel functional $G(x)=\exp \{x(T)\}$ be given. Let $F_{1}(x)=x(T), F_{2}(x)=x^{2}(T)$ and let $F_{3}(x)=\exp \{x(T)\}$. Then using Equations (1) and (5) repeatedly, we have

$$
\begin{aligned}
T_{\gamma, \beta}^{G}\left(F_{1}\right)(y) & =\int_{C_{0}[0, T]}(\gamma x(T)+\beta y(T)) \exp \{x(T)\} d m(x) \\
& =\gamma \int_{C_{0}[0, T]} x(T) \exp \{x(T)\} d m(x)+\beta y(T) \int_{C_{0}[0, T]} \exp \{x(T)\} d m(x) \\
& =\gamma \frac{1}{\sqrt{2 \pi T}} \int_{\mathbb{R}} u \exp \left\{u-\frac{u^{2}}{2 T}\right\} d u+\beta y(T) \frac{1}{\sqrt{2 \pi T}} \int_{\mathbb{R}} \exp \left\{u-\frac{u^{2}}{2 T}\right\} d u \\
& =\gamma T \exp \left\{\frac{T}{2}\right\}+\beta y(T) \exp \left\{\frac{T}{2}\right\} \\
& =(\gamma T+\beta y(T)) \exp \left\{\frac{T}{2}\right\}, \\
T_{\gamma, \beta}^{G}\left(F_{2}\right)(y)= & \int_{C_{0}[0, T]}(\gamma x(T)+\beta y(T))^{2} \exp \{x(T)\} d m(x) \\
= & \int_{C_{0}[0, T]}\left(\gamma^{2} x^{2}(T)+2 \gamma \beta x(T) y(T)+\beta^{2} y^{2}(T)\right) \exp \{x(T)\} d m(x) \\
= & \gamma^{2} \frac{1}{\sqrt{2 \pi T}} \int_{\mathbb{R}} u^{2} \exp \left\{u-\frac{u^{2}}{2 T}\right\} d u+2 \gamma \beta y(T) \frac{1}{\sqrt{2 \pi T}} \int_{\mathbb{R}} u \exp \left\{u-\frac{u^{2}}{2 T}\right\} d u \\
+ & \beta^{2} y^{2}(T) \frac{1}{\sqrt{2 \pi T}} \int_{\mathbb{R}} \exp \left\{u-\frac{u^{2}}{2 T}\right\} d u \\
= & \gamma^{2}\left(T^{2}+T\right) \exp \left\{\frac{T}{2}\right\}+2 \gamma \beta T y(T) \exp \left\{\frac{T}{2}\right\}+\beta^{2} y^{2}(T) \exp \left\{\frac{T}{2}\right\} \\
= & \left(\gamma^{2} T^{2}+\gamma^{2} T+2 \gamma \beta T y(T)+\beta^{2} y^{2}(T)\right) \exp \left\{\frac{T}{2}\right\}
\end{aligned}
$$

and

$$
\begin{aligned}
T_{\gamma, \beta}^{G}\left(F_{3}\right)(y) & =\exp \{\beta y(T)\} \int_{C_{0}[0, T]} \exp \{x(T)\} \exp \{\gamma x(T)\} d m(x) \\
& =\exp \{\beta y(T)\} \frac{1}{\sqrt{2 \pi T}} \int_{\mathbb{R}} \exp \left\{(1+\gamma) u-\frac{u^{2}}{2 T}\right\} d u \\
& =\exp \left\{\beta y(T)+\frac{T(1+\gamma)^{2}}{2}\right\} .
\end{aligned}
$$

While, the formulas for the integral transform $\mathcal{F}_{\gamma, \beta}$ for each functionals $F_{1}, F_{2}$ and $F_{3}$ are given by

$$
\begin{aligned}
\mathcal{F}_{\gamma, \beta}\left(F_{1}\right) & (y)=\int_{C_{0}[0, T]}(\gamma x(T)+\beta y(T)) d m(x)=\beta y(T), \\
\mathcal{F}_{\gamma, \beta}\left(F_{2}\right)(y) & =\int_{C_{0}[0, T]}(\gamma x(T)+\beta y(T))^{2} d m(x) \\
& =\int_{C_{0}[0, T]}\left(\gamma^{2} x(T)+\gamma \beta x(T) y(T)+\beta^{2} y^{2}(T)\right) d m(x) \\
& =\gamma^{2} T+\beta^{2} y^{2}(T)
\end{aligned}
$$

and

$$
\mathcal{F}_{\gamma, \beta}\left(F_{3}\right)(y)=\exp \{\beta y(T)\} \int_{C_{0}[0, T]} \exp \{\gamma x(T)\} d m(x)=\exp \left\{\beta y(T)+\frac{\gamma^{2} T}{2}\right\}
$$




\section{Series Approach of the Generalized Integral Transforms}

In this section, we explain some of the properties of functional analysis theories and establish various formulas for Wiener integrals. We also introduce a new method to evaluate generalized integral transforms using the series expressions.

Let $\mathcal{A} \equiv \mathcal{L}\left(C_{0}[0, T]: \mathbb{R}\right)$ be the class of all bounded linear functionals on $C_{0}[0, T]$ and let $\|\cdot\|_{\mathcal{A}}$ be the norm on $\mathcal{A}$ defined by the formula

$$
\|F\|_{\mathcal{A}}=\sup _{x \in C_{0}[0, T]}|F(x)| .
$$

Also, let $\|\cdot\|_{B V}$ be denote the norm on $B V[0, T]$, the space of all functions of bounded variation on $[0, T]$, defined by the formula

$$
\|w\|_{B V}=|w(0)|+\operatorname{Var}(w)
$$

where, $\operatorname{Var}(w)$ is the total variation of $w \in B V[0, T]$. From the Riesz's Theorem and the Hahn-Banach theorem together with two spaces $\mathcal{A}$ and $B V[0, T]$, we have the following facts:

(i) Every functional $G \in \mathcal{A}$ can be represented by a Riemann-Stieltjes integral

$$
G(x)=\int_{0}^{T} x(t) d w(t)
$$

for some $w_{G} \in B V[0, T]$ with $\operatorname{Var}\left(w_{G}\right)=\|G\|_{\mathcal{A}}$.

(ii) For a nonzero element $x_{0}$ in $C_{0}[0, T]$, there exists a functional $G$ in $\mathcal{A}$ such that $\|G\|_{\mathcal{A}}=1$ and

$$
G\left(x_{0}\right)=\left\|x_{0}\right\|_{C_{0}[0, T]}=\sup _{t \in[0, T]}\left|x_{0}(t)\right| .
$$

(iii) Since $x_{0}(t)=t \in C_{0}[0, T]$, there exists a bounded linear functional $\bar{G}$ such that $\bar{G}\left(x_{0}\right)=$ $\|t\|_{C_{0}[0, T]}=T$. Furthermore, we note that $\bar{G}$ is an element of $\mathcal{A}$ and for some $w_{\bar{G}} \in B V[0, T]$ with $\operatorname{Var}\left(w_{\bar{G}}\right)=\|\bar{G}\|_{\mathcal{A}}=1$,

$$
\int_{0}^{T} t d w_{\bar{G}}(t)=\bar{G}\left(x_{0}\right)=\|t\|_{C_{0}[0, T]}=T .
$$

For a more detailed explanation see [15].

We now explain the importance of our research. Throughout this paper, we always take the functional $G$ to be an element of $\mathcal{A}$, and hence $G(x)=\int_{0}^{T} x(t) d w_{\bar{G}}(t)$ for some $w_{\bar{G}} \in B V[0, T]$ with $\operatorname{Var}\left(w_{\bar{G}}\right)=\|G\|_{\mathcal{A}}$. Let $F_{1}(x)=x(T), F_{2}(x)=\exp \{x(T)\} \equiv e^{F_{1}(x)}, F_{3}(x)=\sin x(T)$ and let $F_{4}(x)=\frac{1}{1+x^{2}(T)}$. Then using Equations (1) and (5), we have

$$
\begin{aligned}
T_{\gamma, \beta}^{G}\left(F_{1}\right)(y) & =\int_{\mathcal{C}_{0}[0, T]}(\gamma x(T)+\beta y(T)) \int_{0}^{T} x(t) d w(t) d m(x) \\
& =\gamma \int_{0}^{t} t d w(t)=\gamma T .
\end{aligned}
$$


and

$$
\begin{aligned}
& T_{\gamma, \beta}^{G}\left(F_{2}\right)(y) \\
& =\int_{C_{0}[0, T]} \exp \{\gamma x(T)+\beta y(T)\} \int_{0}^{T} x(t) d w(t) d m(x) \\
& =\exp \{\beta y(T)\} \int_{0}^{T} \int_{C_{0}[0, T]} x(t) \exp \{\gamma x(T)\} d m(x) d w(t) \\
& =\exp \{\beta y(T)\} \sqrt{\frac{1}{2 \pi t}} \sqrt{\frac{1}{2 \pi(T-t)}} \int_{0}^{T} \int_{\mathbb{R}^{2}} u_{1} \exp \left\{\gamma u_{2}-\frac{u_{1}^{2}}{2 t}-\frac{\left(u_{2}-u_{1}\right)^{2}}{2(T-t)}\right\} d u_{1} d u_{2} d w(t) \\
& =\exp \{\beta y(T)\} \int_{0}^{T} \frac{1}{\pi} \int_{\mathbb{R}^{2}} \sqrt{2 t} v_{1} \exp \left\{\gamma \sqrt{2 t} v_{1}+\gamma \sqrt{2(T-t)} v_{2}-v_{1}^{2}-v_{2}^{2}\right\} d v_{1} d v_{2} d w(t) \\
& =\gamma T \exp \left\{\beta y(T)+\frac{\gamma^{2} T}{2}\right\} .
\end{aligned}
$$

One can see that the second case is more complicated than the first case. Furthermore, we note that the following calculations of $T_{\gamma, \beta}^{G}\left(F_{3}\right)(y)$ and $T_{\gamma, \beta}^{G}\left(F_{4}\right)(y)$ are very tedious or impossible, because the following integrals

$$
\int_{\mathbb{R}^{2}} u_{1} \sin \left(\gamma u_{2}+\beta y(T)\right) \exp \left\{-\frac{u_{1}^{2}}{2 t}-\frac{\left(u_{2}-u_{1}\right)^{2}}{2(T-t)}\right\} d u_{1} d u_{2}
$$

and

$$
\int_{\mathbb{R}^{2}} \frac{u_{1}}{1+\left(\gamma u_{2}+\beta y(T)\right)^{2}} \exp \left\{-\frac{u_{1}^{2}}{2 t}-\frac{\left(u_{2}-u_{1}\right)^{2}}{2(T-t)}\right\} d u_{1} d u_{2}
$$

appear in the calculation of the generalized integral transforms. For this reason, such functionals as $F_{1}$ and $F_{2}$, which are easy to handle, have been considered. In this paper, we will consider a diversity of functionals, including $F_{3}$ and $F_{4}$, which also involve $F_{1}$ and $F_{2}$.

Remark 2. Using the methods in complex variable analysis, the Wiener $T_{\gamma, \beta}^{G}\left(F_{4}\right)(y)$ of $F_{4}$ can be calculated. However, the generalized integral transform $T_{\gamma, \beta}^{G}\left(F_{4}\right)(y)$ is not easy.

Let $\mathbb{C}$ be denote the space of all complex numbers and let

$$
E=\left\{(\gamma, \beta) \in \mathbb{C} \times \mathbb{C}: \gamma^{2}+\beta^{2}=1\right\}
$$

Throughout this paper, we always take a pair $(\gamma, \beta)$ in $E$.

In Lemma 1, we establish the existence of the generalized integral transform.

Lemma 1. Let $G \in \mathcal{A}$ be given, and let $F$ be a functional on $K$. Let $(\gamma, \beta) \in E$. Assume that $\left\{F_{n}\right\}_{n=1}^{\infty}$ is a sequence of functionals on $K$ such that $F_{n} \rightarrow F$ in the sense of $L_{1}\left(C_{0}[0, T]\right)$ as $n \rightarrow \infty$. Then

$$
T_{\gamma, \beta}^{G}\left(F_{n}\right) \rightarrow T_{\gamma, \beta}^{G}(F)
$$

in the sense of $L_{1}\left(C_{0}[0, T]\right)$ as $n \rightarrow \infty$. 
Proof. We note that $G$ is an element of $\mathcal{A}$ and so $|G(x)| \leq M$ for some $M>0$. Using Equation (3), we have

$$
\begin{aligned}
& \int_{C_{0}[0, T]}\left|T_{\gamma, \beta}^{G}(F)(y)-T_{\gamma, \beta}^{G}\left(F_{n}\right)(y)\right| d m(y) \\
& =\int_{C_{0}[0, T]} \mid \int_{C_{0}[0, T]} F(\gamma x+\beta y) G(x) d m(x) \\
& -\int_{C_{0}[0, T]} F_{n}(\gamma x+\beta y) G(x) d m(x) \mid d m(y) \\
& \leq \int_{C_{0}[0, T]} \int_{C_{0}[0, T]}\left|F(\gamma x+\beta y)-F_{n}(\gamma x+\beta y)\right||G(x)| d m(x) d m(y) \\
& \leq M \int_{C_{0}[0, T]} \int_{C_{0}[0, T]}\left|F(\gamma x+\beta y)-F_{n}(\gamma x+\beta y)\right| d m(x) d m(y) \\
& \stackrel{(I)}{=} M \int_{C_{0}[0, T]}\left|F\left(\sqrt{\gamma^{2}+\beta^{2}} z\right)-F_{n}\left(\sqrt{\gamma^{2}+\beta^{2}} z\right)\right| d m(z) \\
& =M \int_{C_{0}[0, T]}\left|F(z)-F_{n}(z)\right| d m(z) .
\end{aligned}
$$

The equality $\stackrel{(I)}{=}$ in Equation (11) follows from the equation

$$
\int_{C_{0}[0, T]} \int_{C_{0}[0, T]} F(p x+q y) d m(x) d m(y)=\int_{\mathcal{C}_{0}[0, T]} F\left(\sqrt{p^{2}+q^{2}} z\right) d m(z)
$$

for a scale-invariant integrable functional $F$, and nonzero complex numbers $p$ and $q$, see $[5,9]$. As $n \rightarrow$ $\infty$, by our assumption, the last expression in (11) tends to zero. Hence we complete the proof of Lemma 1 as desired.

In order to establish Lemma 1 , we assumed that $\left\{F_{n}\right\}_{n=1}^{\infty}$ is a sequence of functionals on $K$ such that $F_{n} \rightarrow F$ in the sense of $L_{1}\left(C_{0}[0, T]\right)$ as $n \rightarrow \infty$. We shall see that a sequence satisfying this condition always exists.

Let $f$ be an infinitely many differentiable function on $\mathbb{R}$ such that

$$
\int_{\mathbb{R}}|f(u)| \exp \left\{-a u^{2}\right\} d u<\infty
$$

for all $a>0$. Then its Maclaurin series is given by the formula

$$
f(u)=\sum_{s=0}^{\infty} \frac{f^{(s)}(0)}{s !} u^{s} \equiv \sum_{s=0}^{\infty} a_{s} h_{s}(u)=\lim _{n \rightarrow \infty} \sum_{s=0}^{n} a_{s} h_{s}(u) \equiv \lim _{n \rightarrow \infty} f_{n}(u)
$$

where $a_{s}=\frac{f^{(s)}(0)}{s !}$ and $f^{(s)}$ is the s-th derivative of $f$.

Remark 3. To apply the Maclaurin series expansion on Euclidean space we have to consider the radius of convergence. The radius of convergence of a series is the radius of the largest disk in which the series converges. It is either a non-negative real number or $\infty$. In Equation (13), we expect that our method can be used for all cases in which the radius of convergence is a non-negative real number or $\infty$.

In our next theorem, we establish that the convergence of a sequence of generalized integral transforms. 
Theorem 2. Let $G \in \mathcal{A}$ be given and let $(\gamma, \beta) \in$ E. For each $n=1,2, \cdots$, let $F_{n}(x)=f_{n}(x(T))$ and let $F(x)=f(x(T))$. Then the generalized integral transform $T_{\gamma, \beta}^{G}(F)$ of $F$ given $G$ is the limit of generalized integral transforms $T_{\gamma, \beta}^{G}\left(F_{n}\right)$ of $F_{n}$ given $G$, namely,

$$
T_{\gamma, \beta}^{G}\left(F_{n}\right) \rightarrow T_{\gamma, \beta}^{G}(F)
$$

in the sense of $L_{1}\left(C_{0}[0, T]\right)$ as $n \rightarrow \infty$, where $f_{n}$ and $f$ are give by Equation (13) above.

Proof. From Lemma 1, it suffices to show that $F_{n} \rightarrow F$ in the sense of $L_{1}\left(C_{0}[0, T]\right)$ as $n \rightarrow \infty$. In order to do this, we note that

$$
\begin{aligned}
L_{n} & \equiv \int_{C_{0}[0, T]}\left|F(x)-F_{n}(x)\right| d m(x) \\
& =\int_{C_{0}[0, T]}\left|f(x(T))-f_{n}(x(T))\right| d m(x) \\
& =\frac{1}{\sqrt{2 \pi T}} \int_{\mathbb{R}}\left|f(u)-f_{n}(u)\right| \exp \left\{-\frac{u^{2}}{2 T}\right\} d u .
\end{aligned}
$$

Now, for each $n=1,2, \cdots$, let

$$
k_{n}(u)=\left|f(u)-f_{n}(u)\right| \exp \left\{-\frac{u^{2}}{2 T}\right\} \equiv\left|f(u)-\sum_{s=0}^{n} h_{s}(u)\right| \exp \left\{-\frac{u^{2}}{2 T}\right\} .
$$

Then $k_{n}$ is nonnegative valued and

$$
\begin{aligned}
\int_{\mathbb{R}}\left|k_{n}(u)\right| d u & \leq \int_{\mathbb{R}}|f(u)| \exp \left\{-\frac{u^{2}}{2 T}\right\} d u \\
& +\int_{\mathbb{R}}\left|f_{n}(u)\right| \exp \left\{-\frac{u^{2}}{2 T}\right\} d u<\infty
\end{aligned}
$$

for all $n=1,2, \cdots$. Hence we can conclude that

$$
\lim _{n \rightarrow \infty} L_{n}=\frac{1}{\sqrt{2 \pi T}} \int_{\mathbb{R}} \lim _{n \rightarrow \infty}\left|f(u)-f_{n}(u)\right| \exp \left\{-\frac{u^{2}}{2 T}\right\} d u=0
$$

which completes the proof of Theorem 2 as desired.

Remark 4. In Lemma 1 and Theorem 2, we considered the case that the radius of convergence is $\infty$. Let $S$ be a proper subset of $\mathbb{R}$ and is Lebesgue measurable which is the radius of convergence. Then we note that

$$
\int_{S}|f(u)| d u \leq \int_{\mathbb{R}}|f(u)| d u
$$

and

$$
\int_{P_{t}^{-1}(S)} F(x) d m(x) \leq \int_{C_{0}[0, T]} F(x) d m(x)
$$

where $P_{t}$ is the projection defined by $P_{t}(x)=x(t)$ from $C_{0}[0, T]$ onto $\mathbb{R}$. From these facts, we can prove all results similar to those in Lemma 1 and Theorem 2 for the case that the radius of convergence is finite.

\section{Series Expression: The Case that the Radius of Convergence is $\infty$}

In this section, we give a new method to calculate generalized integral transform $T_{\gamma, \beta}^{G}(F)$ of various functionals $F$ given $G$. In order to do this, we need two lemmas. The first lemma is the Wiener integration formula. Equation (16) below is obtained from Equations (5) and (8). 
Lemma 2. Let $k$ be a nonnegative integer. Then we have

$$
W_{k} \equiv \int_{0}^{T} \int_{C_{0}[0, T]} x(t) x^{k}(T) d m(x) d w(t)= \begin{cases}0, & k: \text { even } \\ k ! ! T^{\frac{k+1}{2},} & k: \text { odd }\end{cases}
$$

where $k ! !=k \times(k-2) \times(k-3) \times \cdots \times 1$.

The next lemma is the formula for the generalized integral transform.

Lemma 3. Let $(\gamma, \beta) \in E$ and let $G$ be an element of $\mathcal{A}$. For each $n=1,2, \cdots$, let $H_{n}(x)=h_{n}(x(T))$, where $h_{n}$ is as in Equation (13). Then for each $n=1,2, \cdots$, the generalized integral transform $T_{\gamma, \beta}^{G}\left(H_{n}\right)$ of $H_{n}$ given $G$ exists and is given by the formula

$$
T_{\gamma, \beta}^{G}\left(H_{n}\right)(y)=\sum_{l=0}^{\left[\frac{n+1}{2}\right]}{ }_{n} C_{2 l+1}(2 l+1) ! ! \gamma^{2 l+1} \beta^{n-2 l-1} T^{l+1} y^{n-2 l-1}(T)
$$

where $[q]$ is the greatest integer less than equal for a real number $q$.

Proof. We first recall the binomial formula $(a+b)^{n}=\sum_{l=0}^{n}{ }_{n} C_{l} a^{l} b^{n-l}$ with $n=1,2, \cdots$. Using this binomial expansion, Equations (1) and (6), we have

$$
\begin{aligned}
T_{\gamma, \beta}^{G}\left(H_{n}\right)(y) & =\int_{C_{0}[0, T]} H_{n}(\gamma x+\beta y) G(x) d m(x) \\
& =\int_{0}^{T} \int_{C_{0}[0, T]} x(t)[\gamma x(T)+\beta y(T)]^{n} d m(x) d w(t) \\
& =\int_{0}^{T} \int_{C_{0}[0, T]} x(t)\left[\sum_{l=0}^{n}{ }_{n} C_{l}(\gamma x(T))^{l}(\beta y(T))^{n-l}\right] d m(x) d w(t) \\
& =\sum_{l=0}^{n}{ }_{n} C_{l} \gamma^{l} \beta^{n-l} y^{n-l}(T) \int_{0}^{T} \int_{C_{0}[0, T]} x(t) x^{l}(T) d m(x) d w(t) .
\end{aligned}
$$

Using Equation (16), we have that

$$
T_{\gamma, \beta}^{G}\left(H_{n}\right)(y)=\sum_{l=0}^{n}{ }_{n} C_{l} \gamma^{l} \beta^{n-l} y^{n-l}(T) W_{l}
$$

Finally, we obtain Equation (17) by the rearrangement of a sequence in Equation (18) as desired.

From Lemma 3, we can see some observations. Let $G$ be an element of $\mathcal{A}$ and let $H_{n}(x)=h_{n}((T))$ for each $n=1,2, \cdots, 7$. Then we have the following table.

In fact, we can verify all formulas in Table 1 directly. For example, since $G \in \mathcal{A}$, we can write that

$$
G(x)=\int_{0}^{T} x(t) d w(t)
$$


where $w \in B V[0, T]$ and $\operatorname{Var}(w)=\|G\|_{\mathcal{A}}$. Using Equations (5) and (6), we have

$$
\begin{aligned}
& T_{\gamma, \beta}^{G}\left(H_{3}\right)(y) \\
& =\int_{C_{0}[0, T]} \int_{0}^{T} x(t) d w(t)(\gamma x(T)+\beta y(T))^{3} d m(x) \\
& =\int_{0}^{T} \int_{C_{0}[0, T]}\left[\gamma^{3} x(t) x^{3}(T)+3 \gamma^{2} \beta x(t) x^{2}(T) y(T)\right. \\
& \left.\quad+3 \gamma \beta^{2} x(t) x(T) y^{2}(T)+\beta^{3} x(t) y^{3}(T)\right] d m(x) d w(t) \\
& =3 \gamma^{3} T \int_{0}^{T} t d w(t)+3 \gamma \beta^{2} y^{2}(T) \int_{0}^{T} t d w(t) .
\end{aligned}
$$

The last equality in Equation (19) is obtained by the formula

$$
\int_{C_{0}[0, T]} x(t) d m(x)=\int_{C_{0}[0, T]} x(t) x^{2}(T) d m(x)=0 .
$$

Using Equation (8), we have

$$
T_{\gamma, \beta}^{G}\left(H_{3}\right)(y)=3 \gamma^{3} T^{2}+3 \gamma \beta^{2} T y^{2}(T) .
$$

Table 1. Formulas for the polynomial functionals.

\begin{tabular}{lll}
\hline $\boldsymbol{n}$ & $\boldsymbol{H}_{\boldsymbol{n}}(\boldsymbol{x})$ & $\boldsymbol{T}_{\gamma, \boldsymbol{\beta}}^{G}\left(\boldsymbol{H}_{\boldsymbol{n}}\right)(y)$ \\
\hline 1 & $x^{1}(T)$ & $\gamma T$ \\
\hline 2 & $x^{2}(T)$ & $2 \gamma \beta T y(T)$ \\
\hline 3 & $x^{3}(T)$ & $3 \gamma^{3} T^{2}+3 \gamma \beta^{2} T y^{2}(T)$ \\
\hline 4 & $x^{4}(T)$ & $12 \gamma^{3} \beta T^{2} y(T)+4 \gamma \beta^{3} T y^{3}(T)$ \\
\hline 5 & $x^{5}(T)$ & $15 \gamma^{5} T^{3}+30 \gamma^{3} \beta^{2} T^{2} y^{2}(T)+5 \gamma \beta^{4} T y^{4}(T)$ \\
\hline 6 & $x^{6}(T)$ & $90 \gamma^{5} \beta T^{3} y(T)+60 \gamma^{3} \beta^{3} T^{2} y^{3}(T)+6 \gamma \beta^{5} T y^{5}(T)$ \\
\hline 7 & $x^{7}(T)$ & $105 \gamma^{7} T^{4}+315 \gamma^{5} \beta^{2} T^{3} y^{2}(T)+105 \gamma^{3} \beta^{4} T^{2} y^{4}(T)+7 \gamma \beta^{6} T y^{6}(T)$ \\
\hline
\end{tabular}

Remark 5. We can obtain the other expressions of Equation (17) as below: Using the program Mathematica, we have

$$
T_{\gamma, \beta}^{G}\left(H_{n}\right)(y)=\sum_{l=0}^{n}{ }_{n} C_{l} \gamma^{l} \beta^{n-l} \int_{0}^{T} S_{n}(t, l) d w(t)
$$

where

$$
\begin{aligned}
S_{n}(t, l) & =\left(\frac{1-(-1)^{l+1}}{2}\right)\left[\sqrt{2 t \pi}\left(\frac{T}{t}\right)^{\frac{2 k+1}{2}} k !\right. \\
& \left.-\sqrt{2 T} k !\left(\frac{T-t}{t}\right)^{\frac{2 k+1}{2}}{ }_{2} \mathcal{F}_{1}\left[1,-\frac{2 k+1}{2}, \frac{1}{2},-\frac{1}{T-t}\right]\right],
\end{aligned}
$$

$k=\frac{l+1}{2}$ and ${ }_{2} \mathcal{F}_{1}$ is the hyper-geometric function defined by the formula

$$
{ }_{2} \mathcal{F}_{1}[a, b, c, d]=\sum_{m=0}^{\infty} \frac{(a)_{m}(b)_{m}}{(c)_{m}} \frac{d^{m}}{n !},
$$


where

$$
(q)_{m}=\left\{\begin{array}{l}
1, m=0 \\
q(q+1) \cdots(q+m-1), m>0
\end{array} .\right.
$$

In Theorem 3, we establish the series expression of the generalized integral transform.

Theorem 3. Let $(\gamma, \beta) \in E$ and let $G$ be an element of $\mathcal{A}$. For each $n=1,2, \cdots$, let $f, h_{n}$ and $f_{n}$ be as in Equation (13) above. Let $F(x)=f(x(T)), H_{n}(x)=h_{n}(x(T))$ and let $F_{n}(x)=f_{n}(x(T))=\sum_{s=0}^{n} \frac{f^{(s)}(0)}{s !} H_{s}(x)$. Then the generalized integral transform $T_{\gamma, \beta}^{G}(F)$ of $F$ given $G$ exists and is given by the formula

$$
T_{\gamma, \beta}^{G}(F)(y)=\lim _{n \rightarrow \infty} T_{\gamma, \beta}^{G}\left(F_{n}\right)(y)=\lim _{n \rightarrow \infty} \sum_{s=0}^{n} \frac{f^{(s)}(0)}{s !} T_{\gamma, \beta}^{G}\left(H_{s}\right)(y)
$$

in the sense of $L_{1}\left(C_{0}[0, T]\right)$, where

$$
T_{\gamma, \beta}^{G}\left(H_{s}\right)(y)=\sum_{l=0}^{\left[\frac{s+1}{2}\right]}{ }_{s} C_{2 l+1}(2 l+1) ! ! \gamma^{2 l+1} \beta^{s-2 l-1} T^{l+1} y^{s-2 l-1}(T) .
$$

Proof. We note that

$$
\begin{aligned}
T_{\gamma, \beta}^{G}\left(F_{n}\right)(y) & =\int_{C_{0}[0, T]} F_{n}(\gamma x+\beta y) G(x) d m(x) \\
& =\int_{C_{0}[0, T]} \sum_{s=0}^{n} \frac{f^{(s)}(0)}{s !} H_{s}(\gamma x+\beta y) G(x) d m(x) \\
& =\sum_{s=0}^{n} \frac{f^{(s)}(0)}{s !} \int_{C_{0}[0, T]} H_{s}(\gamma x+\beta y) G(x) d m(x) \\
& =\sum_{s=0}^{n} \frac{f^{(s)}(0)}{s !} T_{\gamma, \beta}^{G}\left(H_{s}\right)(y) .
\end{aligned}
$$

From Lemma 3 and Theorem 2, we complete the proof of Theorem 3.

Remark 6. In [16-20], the authors studied the series expansions of appropriate functionals which is called the Ito-Wiener-Chaos expansion. Our method introduced in Theorem 3 is also a kind of these expansions. However, our method is rather useful for evaluating more specific formulas with respect to generalized integral transform.

We give some examples of the series expressions of generalized integral transforms with respect to various functionals that have a radius of convergence of $\infty$.

Example 2. Let $f(u)=\exp \{u\}$. For each $n=1,2, \cdots$, let $f_{n}(u)=\sum_{s=0}^{n} \frac{1}{s !} u^{s}$. Also, let $F(x)=f(x(T))$ and $F_{n}(x)=f_{n}(x(T))$. Then one can easily check that $F_{n} \rightarrow F$ in the sense of $L_{1}\left(C_{0}[0, T]\right)$. Furthermore, we have

$$
F(x)=\exp \{x(T)\}=\sum_{s=0}^{\infty} \frac{1}{s !} x^{s}(T)
$$

and hence using Equation (20) we have

$$
\mathcal{T}_{\gamma, \beta}^{G}(F)(y)=\lim _{n \rightarrow \infty} \sum_{s=0}^{n} \frac{1}{s !}\left(\sum_{l=0}^{\left[\frac{s+1}{2}\right]}{ }_{s} C_{2 l+1}(2 l+1) ! ! \gamma^{2 l+1} \beta^{s-2 l-1} T^{l+1} y^{s-2 l-1}(T)\right) .
$$


Example 3. Let $f(u)=\exp \left\{-u^{2}\right\}$. For each $n=1,2, \cdots$, let $f_{n}(u)=\sum_{s=0}^{n} \frac{(-1)^{s}}{s !} u^{2 s}$. Also, let $F(x)=$ $f(x(T))$ and $F_{n}(x)=f_{n}(x(T))$. Then one can easily check that $F_{n} \rightarrow F_{n}$ in the sense of $L_{1}\left(C_{0}[0, T]\right)$. Furthermore, we have

$$
F(x)=\exp \left\{-x^{2}(T)\right\}=\sum_{s=0}^{\infty} \frac{(-1)^{s}}{s !} x^{2 s}(T)
$$

and hence using Equation (20) we have

$$
\mathcal{T}_{\gamma, \beta}^{G}(F)(y)=\lim _{n \rightarrow \infty} \sum_{s=0}^{n} \frac{(-1)^{s}}{s !}\left(\sum_{l=0}^{\left[\frac{2 s+1}{2}\right]} 2 s+1 C_{2 l+1}(2 l+1) ! ! \gamma^{2 l+1} \beta^{2 s-2 l} T^{l+1} y^{2 s-2 l}(T)\right) .
$$

The following example is to explain the usefulness of the formulas and results mentioned in Section 1 above.

Example 4. Let $f(u)=\sin u$. For each $n=1,2, \cdots$, let $f_{n}(u)=\sum_{s=0}^{n} \frac{(-1)^{s}}{(2 s+1) !} u^{2 s+1}$. Also, let $F(x)=$ $f(x(T))$ and $F_{n}(x)=f_{n}(x(T))$. Then one can easily check that $F_{n} \stackrel{s=0}{\rightarrow} F_{n}$ in the sense of $L_{1}\left(C_{0}[0, T]\right)$. Furthermore, we have

$$
F(x)=\sin x(T)=\sum_{s=0}^{\infty} \frac{(-1)^{s}}{(2 s+1) !} x^{2 s+1}(T)
$$

and hence using Equation (20)

$$
\mathcal{T}_{\gamma, \beta}^{G}(F)(y)=\lim _{n \rightarrow \infty} \sum_{s=0}^{n} \frac{(-1)^{s}}{(2 s+1) !}\left(\sum_{l=0}^{s+1} 2 s+2 C_{2 l+1}(2 l+1) ! ! \gamma^{2 l+1} \beta^{2 s-2 l+1} T^{l+1} y^{2 s-2 l+1}(T)\right) .
$$

Remark 7. We only considered three functionals, however, various functionals that satisfy all the conditions described in the previous sections can be obtained via the series expressions.

\section{Series Expression: The Case that the Radius of Convergence Is Finite}

In this section we give the series expression of the generalized integral transform in the case that radius of convergence is a proper subset of $\mathbb{R}$. The most formulas in the section are obtained from the program Mathematica.

We consider the following functions on $\mathbb{R}$. For $\alpha>1$, let $b(u)=\frac{\sin u^{2}}{u^{2}}, c(u)=(1+u)^{\alpha}, p(u)=$ $\ln (1-u)$ and let $r(u)=\frac{1}{1+u^{2}}$. We now shall give the series expression of the generalized integral transform for each functionals. In order to do this, we need the following concepts. Let $M$ be an any interval in $\mathbb{R}$. Let $\mathcal{P}_{T}: C_{0}[0, T] \rightarrow \mathbb{R}$ be the projection map defined by the formula $\mathcal{P}_{T}(x)=x(T)$. Then $I \equiv \mathcal{P}_{T}^{-1}(M)$ is a Wiener measurable set and for $F(x)=f(x(T))$

$$
\int_{I} F(x) d m(x)=\int_{\mathcal{C}_{0}[0, T]} \chi_{I}(x) F(x) d m(x)=\frac{1}{\sqrt{2 \pi T}} \int_{M} f(u) \exp \left\{-\frac{u^{2}}{2 T}\right\} d u .
$$

Furthermore, for $I^{\prime} \equiv\{x:-\infty<x(t)<\infty, x(T) \in M\}$, we have

$$
\begin{aligned}
& \int_{I^{\prime}} x(t) F(x) d m(x) \\
& =\int_{C_{0}[0, T]} \chi_{I^{\prime}}(x) x(t) F(x) d m(x) \\
& =\frac{1}{\sqrt{2 \pi t}} \frac{1}{\sqrt{2 \pi(T-t)}} \int_{\mathbb{R}} \int_{M} u_{1} f\left(u_{2}\right) \exp \left\{-\frac{u_{1}^{2}}{2 t}-\frac{\left(u_{2}-u_{1}\right)^{2}}{2(T-t)}\right\} d u_{1} d u_{2} .
\end{aligned}
$$

1: The functional $\frac{\sin x^{2}(T)}{x^{2}(T)}$ 
From Theorem 8 and Remark 4, we see that

$$
B(x) \equiv \frac{\sin x^{2}(T)}{x^{2}(T)}=\sum_{s=0}^{\infty} \frac{(-1)^{s}}{(2 s+1) !} x^{4 s}(T), \quad x(T)>0 .
$$

For each $s=0,1,2, \cdots$, let $H_{s}(x)=x^{4 s}(T)$. Then

$$
\begin{aligned}
& \mathcal{T}_{\gamma, \beta}^{G}\left(H_{s}\right)(y) \\
& =\int_{0}^{T} \int_{C_{0}[0, T]} x(t)(\gamma x(T)+\beta y(T))^{4 s} d m(x) d w(t) \\
& =\sum_{l=0}^{4 s}{ }_{4 s} C_{l} \gamma^{l} \beta^{4 s-l} y^{4 s-l}(T) \int_{0}^{T} \int_{C_{0}[0, T]} x(t) x^{l}(T) d m(x) d w(t) \\
& =\sum_{l=0}^{4 s}{ }_{4 s} C_{l} \gamma^{l} \beta^{4 s-l} y^{4 s-l}(T) \\
& \times \int_{0}^{T} \frac{1}{\sqrt{2 \pi t}} \frac{1}{\sqrt{2 \pi(T-t)}} \int_{\mathbb{R}} \int_{0}^{\infty} u_{1} u_{2}^{l} \exp \left\{-\frac{u_{1}^{2}}{2 t}-\frac{\left(u_{2}-u_{1}\right)^{2}}{2(T-t)}\right\} d u_{2} d u_{1} d w(t) \\
& =\sum_{l=0}^{4 s}{ }_{4 s} C_{l} \gamma^{l} \beta^{4 s-l} y^{4 s-l}(T) \int_{0}^{T} \frac{1}{\sqrt{2 \pi t}} \frac{1}{\sqrt{2 \pi(T-t)}}\left[-2^{-\frac{l+2}{2}}(T-t)^{\frac{l}{2}} \Gamma(l+1)\right] d w(t) \\
& =-\sum_{l=0}^{4 s}{ }_{4 s} C_{l} \frac{l !}{\pi} 2^{-\frac{l+4}{2}} \gamma^{l} \beta^{4 s-l} y^{4 s-l}(T) \int_{0}^{T} \frac{(T-t)^{\frac{l-1}{2}}}{\sqrt{t}} d w(t) .
\end{aligned}
$$

Hence using Equation (21), we have

$$
\mathcal{T}_{\gamma, \beta}^{G}(B)(y)=\sum_{s=0}^{\infty} \frac{(-1)^{s+1}}{(2 s+1) !}\left[\sum_{l=0}^{4 s}{ }_{4 s} C_{l} \frac{l !}{\pi} 2^{-\frac{l+4}{2}} \gamma^{l} \beta^{4 s-l} \int_{0}^{T} \frac{(T-t)^{\frac{l-1}{2}}}{\sqrt{t}} d w(t) y^{4 s-l}(T)\right]
$$

in the sense of $L_{1}$.

2: The functional $(1+x(T))^{\alpha}$ for $\alpha>1$

From Theorem 8 and Remark 4 , we see that

$$
C(x) \equiv(1+x(T))^{\alpha}=\sum_{s=0}^{\infty}{ }_{\alpha} C_{s} x^{s}(T), \quad|x(T)|<1
$$


For each $s=0,1,2, \cdots$, let $H_{\mathcal{S}}(x)=x^{\mathcal{S}}(T)$. Then

$$
\begin{aligned}
& \mathcal{T}_{\gamma, \beta}^{G}\left(H_{s}\right)(y) \\
& =\int_{0}^{T} \int_{C_{0}[0, T]} x(t)(\gamma x(T)+\beta y(T))^{s} d m(x) d w(t) \\
& =\sum_{l=0}^{s}{ }_{s} C_{l} \gamma^{l} \beta^{s-l} y^{s-l}(T) \int_{0}^{T} \int_{C_{0}[0, T]} x(t) x^{l}(T) d m(x) d w(t) \\
& =\sum_{l=0}^{s}{ }_{s} C_{l} \gamma^{l} \beta^{s-l} y^{s-l}(T) \\
& \times \int_{0}^{T} \frac{1}{\sqrt{2 \pi t}} \frac{1}{\sqrt{2 \pi(T-t)}} \int_{\mathbb{R}} \int_{-1}^{1} u_{1} u_{2}^{l} \exp \left\{-\frac{u_{1}^{2}}{2 t}-\frac{\left(u_{2}-u_{1}\right)^{2}}{2(T-t)}\right\} d u_{2} d u_{1} d w(t) \\
& =\sum_{l=0}^{s}{ }_{s} C_{l} \gamma^{l} \beta^{s-l} y^{s-l}(T) \\
& \times\left(-\frac{1}{\sqrt{\pi}}(2 T)^{\frac{l-1}{2}}(1+(-1))^{l}\left(\Gamma\left(\frac{l+2}{2}\right)-\Gamma\left(1+\frac{l}{2}, \frac{1}{2 T}\right)\right)\right) \int_{0}^{T} t d w(t) \\
& =-\frac{1}{\sqrt{\pi}} \sum_{l=0}^{\left[\frac{s+1}{2}\right]}{ }_{s} C_{2 l} \gamma^{l} \beta^{s-2 l} y^{s-2 l}(T)\left(2^{\frac{2 l-1}{2}} T^{\frac{2 l+1}{2}}\left(\Gamma\left(\frac{2 l+2}{2}\right)-\Gamma\left(1+\frac{2 l}{2}, \frac{1}{2 T}\right)\right)\right),
\end{aligned}
$$

where $\Gamma(a)$ is the Gamma function and $\Gamma(a, z)$ is the upper incomplete gamma function defined by the formula

$$
\Gamma(a, z)=\int_{z}^{\infty} t^{a-1} e^{-t} d t
$$

and $\Gamma(a, 0)=\Gamma(a)$. Hence, using Equation (22), we have

$$
\begin{aligned}
& \mathcal{T}_{\gamma, \beta}^{G}(C)(y) \\
& =-\sum_{s=0}^{\infty} \frac{{ }_{\alpha} C_{s}}{\sqrt{\pi}}\left[\sum_{l=0}^{\left[\frac{s+1}{2}\right]}{ }_{s} C_{2 l} \gamma^{l} \beta^{s-2 l}\left(2^{\frac{2 l-1}{2}} T^{\frac{2 l+1}{2}}\left(\Gamma\left(\frac{2 l+2}{2}\right)-\Gamma\left(1+\frac{2 l}{2}, \frac{1}{2 T}\right)\right)\right) y^{s-2 l}(T)\right]
\end{aligned}
$$

in the sense of $L_{1}$.

3: The functional $\ln (1-x(T))$

From Theorem 8 and Remark 4, we see that

$$
P(x) \equiv \ln (1-x(T))=-\sum_{s=0}^{\infty} \frac{1}{s} x^{s}(T), \quad|x(T)|<1 .
$$

Using the similar methods in the second case, we can conclude that

$$
\begin{aligned}
& \mathcal{T}_{\gamma, \beta}^{G}(P)(y) \\
& =\sum_{s=0}^{\infty} \frac{1}{s \sqrt{\pi}}\left[\sum_{l=0}^{\left[\frac{s+1}{2}\right]}{ }_{s} C_{2 l} \gamma^{l} \beta^{s-2 l}\left(2^{\frac{2 l-1}{2}} T^{\frac{2 l+1}{2}}\left(\Gamma\left(\frac{2 l+2}{2}\right)-\Gamma\left(1+\frac{2 l}{2}, \frac{1}{2 T}\right)\right)\right) y^{s-2 l}(T)\right]
\end{aligned}
$$

in the sense of $L_{1}$.

4: The functional $\frac{1}{1+x^{2}(T)}$

From Theorem 8 and Remark 4, we see that

$$
R(x) \equiv \frac{1}{1+x^{2}(T)}=\sum_{s=0}^{\infty} \frac{(-1)^{s}}{(2 s) !} x^{2 s}(T), \quad x^{2}(T)<1 .
$$


Using the similar methods in the first and the second cases, we can conclude that

$$
\begin{aligned}
& \mathcal{T}_{\gamma, \beta}^{G}(R)(y) \\
& =\sum_{s=0}^{\infty} \frac{(-1)^{s+1}}{(2 s) ! \sqrt{\pi}}\left[\sum_{l=0}^{\left[\frac{2 s+1}{2}\right]} 2{ }_{2 s} C_{2 l} \gamma^{l} \beta^{2 s-2 l}\left(2^{\frac{2 l-1}{2}} T^{\frac{2 l+1}{2}}\left(\Gamma\left(\frac{2 l+2}{2}\right)-\Gamma\left(1+\frac{2 l}{2}, \frac{1}{2 T}\right)\right)\right) y^{2 s-2 l}(T)\right]
\end{aligned}
$$

in the sense of $L_{1}$.

5: The functional $\frac{1}{\sqrt{4-x(T)}}$

From Theorem 8 and Remark 4, we note that

$$
K(x) \equiv \frac{\sin x^{2}(T)}{x^{2}(T)}=\frac{1}{2} \sum_{s=0}^{\infty}\left(-\frac{1}{4}\right)^{s}{ }_{-\frac{1}{2}} C_{s} x^{s}(T), \quad-4<x(T)<4 .
$$

For each $s=0,1,2, \cdots$, let $H_{s}(x)=x^{\mathcal{S}}(T)$. Then

$$
\begin{aligned}
& \mathcal{T}_{\gamma, \beta}^{G}\left(H_{s}\right)(y) \\
& =\int_{0}^{T} \int_{C_{0}[0, T]} x(t)(\gamma x(T)+\beta y(T))^{s} d m(x) d w(t) \\
& =\sum_{l=0}^{s}{ }_{s} C_{l} \gamma^{l} \beta^{s-l} y^{s-l}(T) \int_{0}^{T} \int_{C_{0}[0, T]} x(t) x^{l}(T) d m(x) d w(t) \\
& =\sum_{l=0}^{s}{ }_{s} C_{l} \gamma^{l} \beta^{s-l} y^{s-l}(T) \\
& \times \int_{0}^{T} \frac{1}{\sqrt{2 \pi t}} \frac{1}{\sqrt{2 \pi(T-t)}} \int_{\mathbb{R}} \int_{-4}^{4} u_{1} u_{2}^{l} \exp \left\{-\frac{u_{1}^{2}}{2 t}-\frac{\left(u_{2}-u_{1}\right)^{2}}{2(T-t)}\right\} d u_{2} d u_{1} d w(t) \\
& =\sum_{l=0}^{s}{ }_{s} C_{l} \gamma^{l} \beta^{s-l} y^{s-l}(T) \\
& \times\left(-\frac{1}{\sqrt{\pi}}(2 T)^{\frac{l-1}{2}}(1+(-1))^{l}\left(\Gamma\left(\frac{l+2}{2}\right)-\Gamma\left(1+\frac{l}{2}, \frac{8}{T}\right)\right)\right) \int_{0}^{T} t d w(t) \\
& =-\frac{1}{\sqrt{\pi}} \sum_{l=0}^{\left.\frac{s+1}{2}\right]}{ }_{s} C_{2 l} \gamma^{l} \beta^{s-2 l} y^{s-2 l}(T)\left(2^{\frac{2 l-1}{2}} T^{\frac{2 l+1}{2}}\left(\Gamma\left(\frac{2 l+2}{2}\right)-\Gamma\left(1+\frac{2 l}{2}, \frac{8}{T}\right)\right)\right)
\end{aligned}
$$

Hence using Equation (23), we have

$$
\mathcal{T}_{\gamma, \beta}^{G}(K)(y)=\frac{1}{2} \sum_{s=0}^{\infty}\left(\frac{1}{4}\right)^{s}{ }_{-\frac{1}{2}} C_{s}\left[\sum_{l=0}^{4 s}{ }_{4 s} C_{l} \frac{l !}{\pi} 2^{-\frac{l+4}{2}} \gamma^{l} \beta^{4 s-l} \int_{0}^{T} \frac{(T-t)^{\frac{l-1}{2}}}{\sqrt{t}} d w(t) y^{4 s-l}(T)\right]
$$

in the sense of $L_{1}$.

We finish this section by giving a remark for possible results and formulas.

Remark 8. (1) We can obtain the series expressions of the generalized integral transform with various radius of convergence.

(2) Under appropriate conditions, we can obtain the following formulas.

$$
\lim _{n \rightarrow \infty} \mathcal{T}_{\gamma_{n}, \beta_{n}}^{G}(F)(y)=\mathcal{T}_{\gamma, \beta}^{G}(F)(y)
$$

and

$$
\lim _{n \rightarrow \infty} \mathcal{T}_{\gamma, \beta}^{G_{n}}(F)(y)=\mathcal{T}_{\gamma, \beta}^{G}(F)(y)
$$


Hence we have

$$
\lim _{n \rightarrow \infty, k \rightarrow \infty} \mathcal{T}_{\gamma_{n}, \beta_{n}}^{G_{k}}(F)(y)=\mathcal{T}_{\gamma, \beta}^{G}(F)(y)
$$

\section{Conclusions}

\subsection{Importance of Results and Formulas in this Paper}

As mentioned in Section 1, all functionals can not be calculated via the change of variable formula on Wiener space. There have been many challenges to solve this problem. However, in our paper, we presented a method to solve the difficulties through series expressions.

Furthermore, we defined the generalized integral transform $\mathcal{T}_{\gamma, \beta}^{G}$. This transform is a more generalized version of the integral transform $\mathcal{F}_{\gamma, \beta}$ introduced by Lee [1]. One can see that if we take $G=1$, then $\mathcal{T}_{\gamma, \beta}^{1}=\mathcal{F}_{\gamma, \beta}$, and this tells us that all formulas in previous papers [3-12] are corollaries of our results. Using the methods on Euclidean space, we established the series expression of the generalized integral transform $\mathcal{T}_{\gamma, \beta}^{G}$. The greatest advantage of this is to evaluate various functionals that cannot be calculated by conventional methods.

\subsection{Expected Results}

We have tried to establish the inverse transform of $\mathcal{T}_{\gamma, \beta}^{G}$. Unfortunately, the existence of the inverse transform has not yet been seen. However, like the research team, we are currently studying the existence and properties of inverse transform, and we expect to establish good results.

Funding: This research received no external funding.

Acknowledgments: The author would like to express gratitude to the referees for their valuable comments and suggestions, which have improved the original paper. This research was supported by Basic Science Research Program through the National Research Foundation of Korea (NRF) funded by the Ministry of Science, ICT and Future Planning (2017R1E1A1A03070041).

Conflicts of Interest: The author declares no conflict of interest.

\section{References}

1. Lee, Y.J. Integral transforms of analytic functions on abstract Wiener spaces. J. Funct. Anal. 1982, 47, $153-164$. [CrossRef]

2. Chung, H.S. Fundamental formulas for modified generalized integral transforms. Banach J. Math. Anal. 2020, 1-17. [CrossRef]

3. Cameron, R.H. Some examples of Fourier-Wiener transforms of analytic functionals. Duke Math. J. 1945, 12, 485-488. [CrossRef]

4. Chang, K.S.; Kim, B.S.; Yoo, I. Integral transforms and convolution of analytic functionals on abstract Wiener space. Numer. Funct. Anal. Optim. 2000, 21, 97-105.

5. Chang, S.J.; Chung, H.S.; Skoug, D. Convolution products, integral transforms and inverse transforms of functionals in $L_{2}\left(C_{0}[0, T]\right)$. Integral Transforms Spec. Funct. 2010, 21, 143-151. [CrossRef]

6. Chung, D.M.; Li, U.C. Transforms on white noise functionals with their applications to Cauchy problems. Nagoya Math. J. 1997, 147, 1-23. [CrossRef]

7. Chung, H.S.; Tuan, V.K. Generalized integral transforms and convolution products on function space. Integral Transforms Spec. Funct. 2011, 22, 573-586. [CrossRef]

8. Chung, H.S.; Choi, J.G.; Chang, S.J. Conditional integral transforms with related topics on function space. Filomat 2012, 26, 1151-1162. [CrossRef]

9. Chung, H.S.; Skoug, D.; Chang, S.J. A Fubini theorem for integral transforms and convolution products. Int. J. Math. 2013, 24, 1350024. [CrossRef]

10. Im, M.K.; Ji, U.C.; Park, Y.J. Relations among the first variation, the convolutions and the generalized Fourier-Gauss transforms. Bull. Korean Math. Soc. 2011, 48, 291-302. [CrossRef]

11. Kim, B.J.; Kim, B.S.; Skoug, D. Integral transforms, convolution products and first variations. Int. J. Math. Math. Soc. 2004, 11, 579-598. [CrossRef] 
12. Kim, B.S.; Skoug, D. Integral transforms of functionals in $L_{2}\left(C_{0}[0, T]\right)$. Rocky Mt. J. Math. 2003, 33, 1379-1393. [CrossRef]

13. Johnson, G.W.; Skoug, D.L. Scale-invariant measurability in Wiener space. Pac. J. Math. 1979, 83, 157-176. [CrossRef]

14. Arsen, P. Green functions of the first boundary-value problem for a fractional diffusion-Wave Equation in Multidimensional Domains. Mathematics 2020, 8, 464. [CrossRef]

15. Kreyszig, E. Introductory Functional Analysis with Applications; John Wiley and Sons: Hoboken, NJ, USA, 1978.

16. Cao, Y. On Convergence rate of Wiener-Ito expansion for generalized random variables. Int. J. Probab. Stoch. Process. 2006, 78, 179-187. [CrossRef]

17. Hu, Y. Ito-Wiener Chaos Expansion with Exact Residual and Correlation, Variance Inequalities. J. Theor. Probab. 1997, 10, 835-848. [CrossRef]

18. Chenkuan, L.; Kyle, C. Babenkos Approach to Abels Integral Equations. Mathematics 2018, 6, 32. [CrossRef]

19. Zeeshan, K.; Muhammad, A.K.; Saeed, I.; Bilal, J.; Fawad, H.; Haroon, U.R.; Waris, K. Analysis of Magneto-hydrodynamics Flow and Heat Transfer of a Viscoelastic Fluid through Porous Medium in Wire Coating Analysis. Mathematics 2017, 5, 27. [CrossRef]

20. Chih-Hsueh, L.; Guo-Hsin, H.; Jun-Juh, Y. Chaos Suppression in Uncertain Generalized Lorenz? Stenflo Systems via a Single Rippling Controller with Input Nonlinearity. Mathematics 2020, 8, 327. [CrossRef]

(C) 2020 by the author. Licensee MDPI, Basel, Switzerland. This article is an open access article distributed under the terms and conditions of the Creative Commons Attribution (CC BY) license (http://creativecommons.org/licenses/by/4.0/). 